\title{
La cobertura televisiva del fútbol en el Sur de Brasil y de España: RBS - Canal Sur
}

\author{
Joaquín M. Marín Montín \\ Investigador de Comunicación y Deporte. Licenciado en Ciencias de la Información. \\ Universidad de Sevilla. Becario Intercampus en la Universidade Federal de \\ Santa Maria (Brasil)
}

\section{RESUMEN}

Este artículo constituye parte de un trabajo comparativo entre Brasil y España, correspondiente al tratamiento que los medios de comunicación hacen del deporte. En él se analizan las claves que configuran el acercamiento a un mismo fenómeno sociológico, como es el fútbol, en dos realidades culturales diferentes. La programación deportiva de televisión llevada a cabo por Canal Sur en Andalucía y RBS en Rio Grande do Sul, así como la cobertura que ambas cadenas realizan en sus retransmisiones en directo del fútbol completan el análisis del presente estudio.

\section{ABSTRACT}

This article is part of a comparative research between Brazil and Spain, about how the media treats the sports activities. Besides, this study analyses the keys of the same sociological phenomenon, which is football, in two different cultural realities. The broadcasting on television regarding sports events in Andalusia with Canal Sur, and Rio Grande do Sul with RBS. The live broadcasting of these channels completes the analysis of the present research.

Palabras claves: Brasil/España/Deporte/Fútbol/Televisión/Canal Sur/RBS/Andalucía/Rio Grande do Sul.

Key Words: Brazil/Spain/Football/Sport/Football/Television/Canal Sur/RBS/Andalusia/Rio Grande do Sul.

ndalucía y Rio Grande do Sul se enclavan en la zona meridional de dos
países que viven de forma apasionada el fútbol. Los medios de comu-
nicación no podían dejar escapar este fenómeno y especialmente la
televisión, responsable en gran medida de su divulgación. No hay duda de que el fútbol es actualmente el deporte más seguido a través de la pequeña pantalla. En los albores de un nuevo siglo, se ha producido un nuevo fenómeno y es la interdependencia creada entre el medio audiovisual con el fútbol y viceversa. La transformación en estos últimos años de este deporte debido a la venta y explotación de los derechos de televisión de los clubes en las principales compe- 
ticiones ha disparado cifras de dinero inimaginables hasta entonces. Los equipos de fútbol se han convertido en poderosas empresas, que invierten su presupuesto gracias a estos ingresos, generados en gran parte de la televisión.

Las retransmisiones televisivas de eventos deportivos, especialmente de fútbol, ocupan un lugar muy importante en la programación de la mayoría de las televisiones. En el caso de la Radio Televisión Pública de Andalucía no podía ser menos y este hecho ha sido crucial para la consolidación como cadena de grandes audiencias. Tras doce años de historia, Canal Sur ha dado siempre una gran importancia al seguimiento de los principales acontecimientos deportivos, sobre todo aquellos celebrados en Andalucía. Desde el principio se apostó fuerte por el "deporte rey". Durante estos años ha mantenido la exclusiva de la Liga española de Fútbol junto al resto de las televisiones autonómicas. "Buena parte del esfuerzo se centró además en el deporte, con una amplia propuesta que culminaba el domingo con un programa de ocho horas de duración, La Jugada y que presentaba Joaquín Durán. En el programa se intercalaban juegos y concursos, además de la información deportiva". ${ }^{1}$

Los nombres de los espacios deportivos se han referido de una forma u otra a este deporte: La Jugada, El Remate, La Liga, Club deportivo, Gol a Gol, lo que evidencia la importancia que ha tenido y sigue teniendo para la cadena estos programas. Sin embargo no se puede olvidar el tratamiento que Canal Sur ha llevado a cabo del resto de las actividades deportivas especialmente aquellas que se desarrollan en Andalucía además de retransmisiones de deportes poco habituales como el tenis de mesa, el baloncesto femenino o el Motocross. Con la entrada del segundo canal terrestre el 5 de junio de 1998, Canal 2 Andalucía, la oferta deportiva crecerá notablemente en televisión, y donde el deporte será una pieza clave de su programación diaria, sobre todo fines de semana. ${ }^{2}$

Gracias a la puesta en marcha del segundo canal, más enfocado al deporte, se suelen producir en estos últimos tres años una media de veinte retransmisiones deportivas anualmente en Andalucía, entre las que encontramos habitualmente baloncesto, voleibol, balonmano y carrera de caballos. Actividades deportivas en las que haya protagonismo andaluz tendrán preferencia para la cadena como corresponde al ser una empresa pública andaluza. ${ }^{3}$

En cuanto a los programas deportivos que se incluyen actualmente (segundo semestre del 2000 y primero del 2001) en la parrilla de ambos canales:

En primer lugar estarían las retransmisiones en directo del fútbol, los sábados en Canal Sur el partido de segunda división a las 18:30 y posteriormente a las

1 CASTRO, Benito y otros: RTVA, Diez años con Andalucía, Dirección de Imagen y Comunicación RTVA, Sevilla, 1999, p.23.

2 Id., p.75.

3 Libro de estilo de Canal Sur Televisión. RTVA, 1991, p.35. 
21:00 horas bajo el título de La Liga el de primera división. El partido de Fútbol de segunda $B$ en Canal 2 el domingo por la tarde cerraría este bloque de partidos emitidos durante el fin de semana.

El baloncesto de la Liga ACB también es retransmitido por Canal 2, sobre todo de los equipos andaluces que juegan en la máxima competición del baloncesto español ( en la temporada 2000-01 Unicaja de Málaga y Caja San Fernando de Sevilla), así como partidos de carácter internacional como son ligas europeas en la que estén equipos andaluces.

Pero sobre todo, uno de los espacios más importantes en el conjunto de la programación de la televisión pública andaluza es:

Gol a Gol, resume la jornada de la liga de fútbol los domingos de cada semana. Tiene un doble formato ya que se inicia en Canal Sur de 21:00 a 21:45 y continúa una segunda parte más ampliada en Canal 2, de 22:00 a 00:00. Desde el momento que empieza a rodar el balón en los diferentes estadios se va preparando la escaleta y el guión del programa. En diferentes cabinas están los redactores siguiendo los partidos en directo de los equipos de fútbol andaluces, reseñando aquellos momentos más importantes del juego, para posteriormente realizar el montaje del resumen del partido. Tanto el editor del programa, Manuel Ladrón de Guevara como el realizador Juan Valentín Gamazo supervisan todo los preparativos del programa que se iniciará en directo horas después. La primera parte de Gol a Gol presentado por Miguel Ángel Cortes y Victoria Romero está más orientada a mostrar lo más destacado de cada jornada de Liga de la primera y segunda división del fútbol español. La segunda parte del programa emitida en Canal 2 y presentada por Antonio Bustos, analiza más detenidamente la jornada reseñando especialmente lo realizado por los equipos andaluces, además de dar a conocer la crónica de los equipos andaluces en la segunda división B.

Por otro lado, el programa Tododeporte se ha ido emite diariamente en Canal 2 y se ocupa de la actualidad deportiva a modo de informativo en 15 minutos. Los sábados y domingos pasa a emitirse en horario matinal y suele incluir retransmisiones en directo, pasando a denominarse Tododeporte fin de semana. Entre ellas, destacan las competiciones de carrera de caballos, celebradas diferentes lugares, así en la temporada de invierno en el Hipódromo de Pineda en Sevilla, durante la primavera en Mijas y finalmente en verano en la playa de Sanlúcar de Barrameda. El resto de la programación deportiva se completaría con otros programas monográficos de otras modalidades deportivas como Liga ACB dedicado al baloncesto nacional e internacional y recientemente creado el programa A caballo sobre el mundo ecuestre de competición. La política de Canal 2 Andalucía en sus tres años de existencia ha tratado por tanto de dar espacio, además del fútbol, a los más variadas actividades deportivas, destacando especialmente las retransmisiones en directo de los siguientes deportes: 
- Baloncesto, balonmano, voleibol.

- Tenis de mesa, tenis y padel.

- Motocross, supercross y trial indoor.

- Carreras de caballo, saltos hípicos, concursos de acoso y derribo.

- Atletismo, natación.

- Fútbol sala, fútbol siete y fútbol infantil.

- Rugby.

- Deporte aventura.

- Ajedrez.

Si realizamos una análisis comparativo con la cadena brasileña $R B S$, el fútbol es sin duda el contenido esencial en su parrilla de programas deportivos. Hay que partir de la base del significado que este deporte tiene para este enorme país, "paixao maior do povo brasileiro". ${ }_{4}^{4}$ Para Brasil el fútbol constituye una de las principales fuentes de identidad de su nación. El peso histórico de su equipo nacional de haber ganado cuatro Copas del Mundo hasta el momento, la figura de Pelé y tantos jugadores que han dado a conocer el nombre de Brasil por todo el mundo es más que una fuente de orgullo para el todo su pueblo. Debido a ello, este deporte ocupa la mayor parte de los programas informativos y retransmisiones en directo de la televisión.

A diferencia de Andalucía, el grupo RBS constituye una empresa multimedia regional de carácter privado situada al Sur de Brasil. Fundada en 1957 por Mauricio Sirotsky Sobrinho, opera hasta el momento con radio, televisión, periódicos, servicio de información on line, televisión por cable y con una fundación social dirigida a niños y adolescentes. ${ }^{5}$

RBS televisión es una red de televisión filial del grupo Globo, primer grupo de comunicación de Brasil, aunque su gestión empresarial y comercialización es independiente. Cubre dos de los tres estados sureños del país con una red de 17 emisoras que actúa en los estados de Rio Grande do Sul y de Santa Catarina y está presente en todas las microregiones de esos estados. Porto Alegre, capital de Rio Grande do Sul, y Florianapolis, de Santa Catarina, serían los dos grandes centros emisores, de los que a su vez se ramificarían diferentes emisoras. Líder de audiencia en el Sur de Brasil constituye "el principal vehículo de comunicación de los medios electrónicos en el sur del país". ${ }^{6}$

$\mathrm{Al}$ ser una empresa filial del grupo Globo, $R B S$ tiene un orden jerárquico en su programación televisiva:

4 LEVER, Janet: A loucura do futebol, Chicago, Universidad de Chicago (Illanois), 1983, p10.

5 RBS Brasil. "Grupo RBS”.Porto Alegre, 2000, p.1.

6 Id., p.24. 
Nacional (Brasil) - Estadual (Rio Grande do Sul, Santa Catarina) - Local (Por ejemplo Santa María y algunos municipios de su área cercana). El tiempo de programación propia que la Globo da a sus filiales es de 25 minutos diarios. $R B S$ frente a otras filiales del país ocupa el cien por cien del horario ofrecido por Globo.

En cuanto a a su programación deportiva, no hay una regla fija que sustente las retransmisiones deportivas en esta cadena. El deporte altera el reparto de horas que Globo proporciona a $R B S$, concediendo mayor o menor espacio dependiendo del alcance social del momento en que se encuentre una actividad deportiva. Estadísticamente el fútbol constituye el $70 \%$ de la programación deportiva. La audiencia además es la que manda, y si hay interés cubrirá o no determinados eventos deportivos. En este sentido, la ciudad de Caxias do Sul tiene en su programación televisiva de RBS dos bloques, ya que tiene dos equipos de fútbol en las principales competiciones (Juventude, Caxias) frente a otras ciudades en las que RBS tiene apenas un solo bloque.

$\mathrm{Al}$ igual que en el resto de Brasil, habría que delimitar dos grandes universos en la programación deportiva de $R B S$ :

1) El fútbol.

2) Los demás deportes.

1) En el caso del deporte rey, $R B S$ tiene los derechos del Campeonato Gaúcho ${ }^{7}$, así como de su Liga de Ascenso. A nivel nacional estará en función de lo que más interese a la cadena Globo, dependiendo de la trayectoria o momento de los equipos de Rio Grande do Sul (primeras posiciones en la clasificación, partidos de final,...). En este último caso si la transmisión es en abierto, se seguirá una de las normas de la Globo, por la cual la ciudad donde se juegue el partido no podrá verlo. Es decir, si el partido es del Gremio de Porto Alegre contra Flamengo de Rio de Janeiro, se podrá ver en todo el país y Estado, pero no en Porto Alegre. De esta forma se busca una mayor asistencia del público a los estadios.

2) En Rio Grande do Sul, por razones culturales y climáticas (más frío), el fútbol sala (futsal para los brasileños) podría considerarse el segundo deporte de más tradición en el Sur del país. Para la televisión es además un deporte cuya cobertura es más fácil y barata de transmitir, ya que no necesita tanto despliegue de medios técnicos y humanos como el fútbol. Del resto de los deportes, el tratamiento de otros deportes para $R B S$ variará en función del momento en que se encuentren determinadas actividades deportivas. De ahí que no se pueda establecer una jerarquía. A igual que en Canal Sur, para $R B S$ tendrá preferencia actividades deportivas celebradas su área de interés. Ese fue el caso del baloncesto que vivió un excelente momento en Santa Cruz do Sul, cuando su equipo el Corintians fue

7 Campeonato Gaúcho, denominación del Torneo entre equipos de Rio Grande do Sul. 
campeón nacional de Brasil, aumentando la difusión de este deporte considerablemente como nunca hasta entonces se había hecho en este Estado.

El voleibol es otro de los deportes tras el fútbol sala que también es tratado ampliamente tanto en categoría femenina como masculina.

Finalmente estarían en un grupo má minoritario el voley-playa, mountainbike,... en pequeños bloques de tiempo, emitido más por razones de espectacularidad en sus imágenes.

Hay que destacar igualmente dentro de la estructura de la cadena $R B S$ un segundo canal, TVCOM, enfocado a asuntos que interesan a una comunidad más local. Pertenece exclusivamente a $R B S$ y no depende de Globo. Su área de cobertura es también Rio Grande do Sul y Santa Catarina. Puede verse en abierto a través de la UHF en Porto Alegre, mientras que en el resto de su área, su difusión se lleva a cabo mediante el cable. Su tiempo de programación en directo es de 8 horas diarias. Podríamos considerarlo como el equivalente de Canal 2 Andalucía, sobre todo por tratar otras disciplinas deportivas de mayor protagonismo en el Sur de Brasil que no son el fútbol.

\section{Normas en las transmisiones del fútbol de Canal Sur y $R B S$}

Tanto Canal Sur como RBS en el Sur de España y Brasil, respectivamente, siguen un patrón previamente adoptado que deben adecuar en las transmisiones deportivas de fútbol.

En el caso de la Radio Televisión Pública Andaluza, al formar parte de FORTA (Federación de Televisiones Autonómicas) emite partidos que intercambia con este grupo de televisiones más TVE en aquellas comunidades autónomas que no tienen televisión propia. Por otro lado, AudioVisual Sport, empresa que gestiona, administra y explota los derechos audiovisuales correspondientes a la Liga Nacional de Fútbol Profesional (Primera y Segunda División del Campeonato español y la Copa del Rey) desde 1998 y hasta el 2003, se encarga de la producción y realización de los encuentros de PPV (pago por visión), definió unos criterios básicos generales de producción para estructurar las distintas señales que las empresas encargadas de dar servicio de la cobertura de la LFP (Liga Nacional de Fútbol española de primera y segunad división), entregan cada jornada de Liga, a Audiovisual Sport.

En el caso brasileño, el fútbol ocupa la mayor parte de los noticiarios y transmisiones en directo de la televisión brasileña, “...No Brasil o futebol é uma das principais fontes de identidade da naçao". ${ }^{8}$

8 PEREIRA, Irany: A cobertura esportiva na televisao, UNISINOS, Sao Leopoldo, 1993, p1. 
Si nos referimos a partidos de fútbol de máximo nivel, como Campeonato nacional (denominado en el 2000 Copa Joao Havelange y el otro gran torneo, la Copa do Brasil, equivalente a la Copa del Rey en España) hay un patrón a seguir y éste no es otro que el que manda la cadena Globo. Ésta tiene exclusividad en la retransmisión de los partidos del Campeonato brasileño, la Globo será la que acordará con los mayores clubes del país. El denominado "Clube dos trece" (mejores 13 equipos) aunque realmente son 16 equipos los que la cadena representa. No existe ninguna empresa, al modo de Audiovisual Sport, que intermedie en las negociaciones de los partidos televisados. Por otro lado, en la "Copa de Brasil" la Rede Globo tiene también exclusividad, negociando directamente con los clubes la retransmisión de sus partidos. Otras cadenas brasileñas como Bandeirantes sólo transmiten la Copa Libertadores de América y la Copa Mercosur, que también retransmite la Globo. Por último, la modalidad de "pago por visión" también la realiza la Globo. Al tratarse de un país de grandes dimensiones y en donde se celebran muchísimos campeonatos simultáneamente, la cobertura del campeonato brasileño sigue un esquema de la cadena Globo, que es la que más partidos produce y emite en diferentes modalidades (televisiones afiliadas, pago por visiónSportv,...). Otras cadenas como la mencionada Bainderantes, intentan desmarcarse de la línea Globo, aunque el número de transmisiones realizadas de fútbol es muy inferior al de aquella.

En el inicio del Campeonato español (Liga y Copa del Rey) normalmente se suelen negociar los partidos que las cadenas ofrecerán. De este forma la Forta negocia con Audiovisual Sport qué partidos le interesa elegir junto a Canal Plus, el resto se ofrecen a través de la modalidad de pago por visión. Sin embargo, esto cambia en el caso de Brasil, sólo unos días antes del partido e incluso el mismo día del juego se sabrá si se emitirá o no en alguna de las modalidades de visión. A veces también ocurre que se anuncie un partido y se vea otro, lo que genera un cierto desorden en la programación televisiva.

\section{Análisis de las retransmisiones de fútbol en Canal Sur}

La cadena pública andaluza de acuerdo con la FORTA produce y realiza aquellos partidos de fútbol que previamente han sido acordados entre las diferentes televisiones. En la realización en directo de un partido de fútbol es importante tener en cuenta para la ubicación de las cámaras el eje por donde transcurre la acción. En este caso es el estadio y "es el que marcan los contendientes en su lucha por llegar a una meta concreta, ya sea la portería del rival, la meta en una carrera, etc.”, como señala Jose María Castillo. ${ }^{9}$ De ahí que en el fútbol la línea de acción

9 CASTILlO, J.M: Elementos del lenguaje audiovisual. IORTV, Madrid, 1997, p.45. 
está bastante bien definida a la hora de emplazar las cámaras, cuyo esquema básico estaría de una portería a otra.

Son tres tipos de cobertura las que lleva a cabo Canal Sur en sus retransmisiones en directo según sea la el partido de fútbol de que se trate:

$2^{\text {a }}$ División B, 2a División y Primera División.

En primer lugar, estaría la $2^{a}$ División B - Grupo IV, compuesta casi exclusivamente por equipos andaluces, los cuatro primeros compiten entre ellos para el ascenso a $2^{\mathrm{a}}$ División. Su emisión comenzó los viernes en directo a las 21:00 horas y posteriormente los domingos a las 19:00 horas. Se lleva a cabo con una Unidad Móvil de cinco cámaras, en diferentes posiciones: Master general, master corto, dos en línea de fuera de juego y una cámara a pie de campo. Esta última empieza al hombro para mostrar la salida de los jugadores al campo, sorteo, entrenadores,... para posteriormente situarse en una plataforma redonda con una silla y un "bazooka" para la copa de la cámara que se sitúa en el centro del campo en la banda. Dicha plataforma recibe coloquialmente el nombre de "peseta".

Para la narración del partido, Canal Sur lleva su narrador de la redacción de deportes, al que le acompañarán un comentarista invitado que tenga que ver con alguno de los dos equipos (ex-jugador o entrenador) y un entrevistador a pie de campo en la zona de banquillos, éste suele ser de la provincia en la que se celebra el partido.

En cuanto a las repeticiones, se cuentan con tres aparatos magnetoscopios VTR: Uno de ellos comparte con una matriz la grabación de los fuera de juego según vaya la dirección del juego, otro VTR graba el master corto y el último la cámara de la "peseta".

La rotulación e inserción de elementos gráficos se realiza desde el centro receptor de Sevilla, donde se introducen además de los rótulos, las numerosas cortinillas y sobre impresiones de publicidad.

Los enlaces con el centro emisor central de Sevilla se realizan de tres maneras, la más común es por fibra óptica que en España está instalada en casi todos los estadios de fútbol, o si no por enlace terrestre con el centro de Canal Sur TV de esa provincia y desde allí por fibra óptica al centro de Sevilla. La tercera opción es vía satélite, contratando una parabólica a Telefónica, utilizando el Satélite Hispasat. El equipo técnico básico de esta retransimisión se compone de: Un realizador, un productor, un mezclador de imágenes, un jefe técnico de Unidad Móvil, un operador de control de cámaras, cinco operadores de cámara, un operador de VTR, un operador de sonido, un técnico de enlaces, cinco montadores, un conductor de la Unidad Móvil y un conductor del grupo electrógeno.

Para partidos de segunda división, el esquema técnico varía sustancialmente. Se realizan con dos ó tres cámaras más, entre 6 y 8 cámaras para las siguientes posiciones: Master general, master corto, dos en líneas de fuera de juego, una "peseta" a la izquierda del banquillo en la banda izquierda, otra igual en la banda derecha, una "peseta" detrás de la portería (que se emplea también para la entrevistas) y una en lo 
alto de la grada izquierda. Se utiliza además un VTR más que maneja todas las cámaras de detrás de las porterías. El equipo técnico humano se incrementa con tres operadores de cámaras más, un operador VTR y dos montadores.

La emisión de este partido es el sábado a las 18:00 horas, y va enmarcado en un programa que engloba un "previo" (lo que sucede antes del juego), el partido, el "previo" del partido de primera división, un informativo de media hora para culminar con el partido de primera. Cuando son muchos los partidos que se juegan los sábados suele haber también otro programa de resúmenes de partidos de 23:00 a 00:00.

La programación del fútbol en Canal Sur incluye un espacio conductor, que se hace en un plató con un gran decorado, lleno de pantallas desde las que se hacen todas las conexiones en directo con los diversos campos en los que transcurre los partidos esa tarde. En el plató, situado en Sevilla, se encuentra un conductorpresentador y dos invitados para el partido de $2^{\mathrm{a}}$ División y dos para el de $1^{\mathrm{a}}$, además se ofrecen resúmenes de partidos anteriores, las noticias de la semana, y conectan en directo con quien está a pie de campo en cada partido.

\section{UBICACIÓN DE CÁMARAS: \\ PARTIDO DE $2^{\text {a }}$ DIVISION \\ PARTIDO BETIS-COMPOSTELA \\ CANAL SUR (FORTA)}
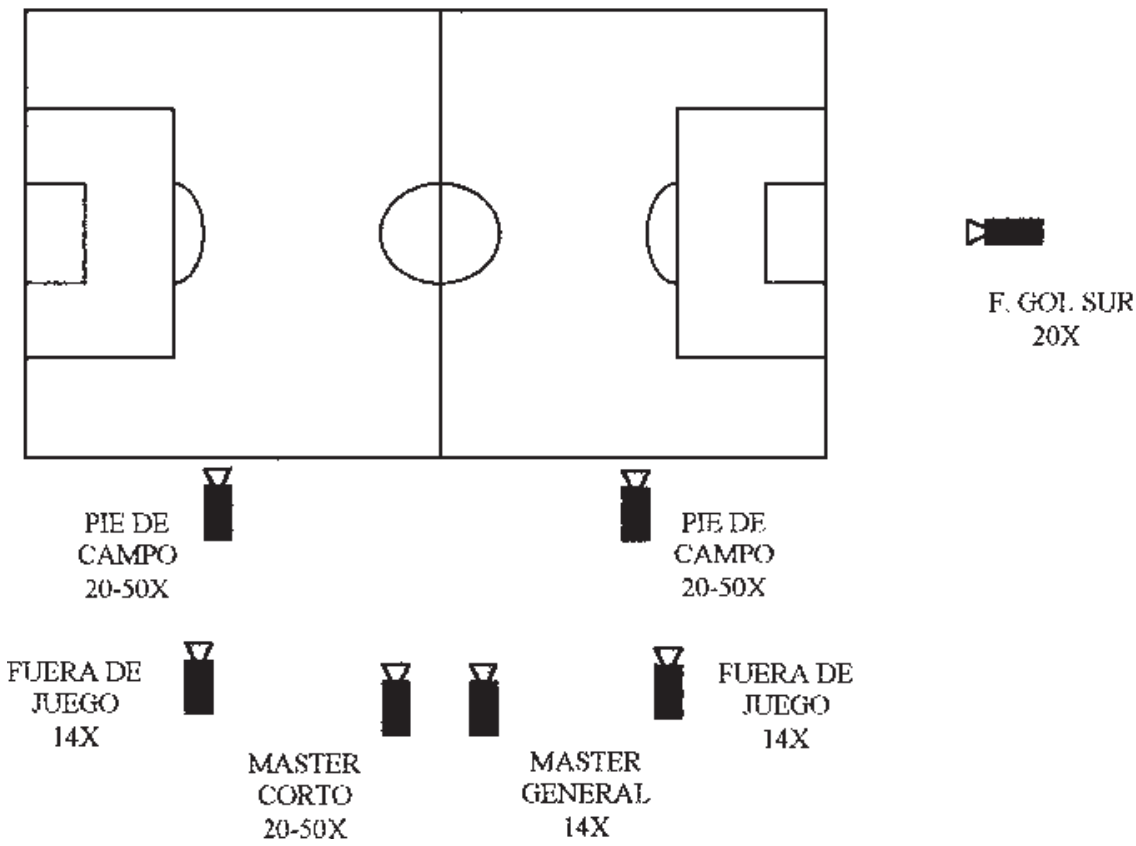
El partido de $1^{\text {a }}$ División de los sábados por la noche culmina la programación deportiva futbolística de Canal Sur. Emitido en hora de máxima audiencia, a las 21:00 horas, y para todas las televisiones regionales que pertenecen a FORTA. El número de cámaras utilizadas aumenta respecto a los dos esquemas anteriores, pasando a utilizarse 9 cámaras, añadiéndose una más en la banda derecha en "peseta". Esta cámara se le denomina "superlenta" y tiene una óptica de 70X, se le da mucha velocidad de obturación para que se vean las repeticiones nítidamente y se graba en un vídeo especial que reproduce cada frame (cuadro) de imagen leyendo dos campos en vez de uno, con lo que se logra una gran nitidez en las repeticiones y ofreciendo muchísimos recursos expresivos a la realización. Esta cámara es propiedad de la FORTA. La retransmisión se elabora en formato digital, con un sistema de repeticiones a través de un disco duro.

Este partido lleva aproximádamente nueve posiciones de comentaristas dobles (narrador y comentarista) correspondientes a las diferentes televisiones autonómicas que pertenecen a FORTA y a TVE, que emite el partido por su segundo canal en aquellas regiones que no tienen canal autonómico.

Además de estos tres modelos de partidos, desde hace dos años se puso en marcha el sistema en PPV (modalidad de pago por visión) a los que Canal Sur ofrece sus servicios tras un acuerdo con Audiovisual Sport, propietaria de los derechos de imagen de los equipos de $1^{\text {a }}$ y $2^{\text {a }}$ división para los partidos que ellos soliciten, pagándole esta producción y a su vez la FORTA paga por emitir un partido de $1^{a}$ división los sábados a las 21:00, que es el único partido de Liga que actualmente se emite en abierto en España. Para el PPV se siguen las normas de Audiovisual Sport, existiendo tres modalidades de producción en función de la importancia del partido ${ }^{10}$ :

La primera opción incluiría doce cámaras y cinco VTR. Además de las habituales se incluyen otras, como una en ángulo inverso de óptica de 20X, otra detrás de la portería izquierda en grúa (normalmente una cabeza caliente con objetivo angular), una detrás de la portería derecha en lo alto de la grada $20 \mathrm{X}$ y una cámara para planos en supergeneral con objetivo angular. La segunda opción sería de ocho cámaras y cuatro VTR y varía poco de la del partido de primera división de los sábados. Por último, estaría la opción básica con cuatro cámaras y 3 VTR.

\section{Análisis de las retransmisiones de fútbol en RBS - Globo}

En el caso del fútbol brasileño, la mayor parte de los partidos que se televisan los produce y realiza la cadena de televisión Globo, que negocia directamente con los clubes de fútbol como se señaló anteriormente. $R B S$, filial del grupo Globo en el Sur de Brasil, lleva a cabo las retransmisiones deportivas que 
se celebran en esta parte del país. Los partidos más importantes son los que corresponden al Campeonato Brasileño (equivalente a la Primera División española) y en el que participan diversos equipos del Sur de Brasil que además se suelen transmitir para todo el país.

Hay que tener en cuenta la arquitectura de la mayoría de los estadios de fútbol en Brasil, la mayoría de ellos suelen tener una pista semicircular que rodea al campo de juego, como una pista de atletismo. Esto influye en la configuración de las retransmisiones, ya que implica un posicionamiento de las cámaras en ángulo más abierto que en los estadios españoles. Hay mayor espacio entre el terreno de juego y el graderío. A modo de ejemplo, analizaremos el clásico Grenal entre los equipos Gremio e Internacional de Porto Alegre. ${ }^{11}$

Este partido fue retransmitido por la cadena Globo, a través de la modalidad de pago por visión, en el canal temático Sportv. ${ }^{12}$

Correspondió a una de las jornadas de la Copa Joao Havelange (Campeonato Brasileño). Se llevó a cabo con una Unidad Móvil de siete cámaras, colacadas en las siguientes posiciones:

Dos cámaras para planos Master general y corto, una de ellas estaba en la cabina donde se encontraba el narrador y el comentarista, otras dos cámaras en posición de línea de fuera de juego aunque ubicadas más en curva, también denominadas “cámaras inglesas”. Detrás de cada portería, aunque más cercana a la tribuna central, estarían otras dos cámaras aunque en posición más abierta y denominadas "cámaras italianas". Por último, una cámara a pie de campo con una óptica mayor 70X "slow motion" (superlenta) cuya señal se graba en un VTR digital, con las mismas características que la utilizada por Canal Sur y la Forta en los partidos de $1^{\mathrm{a}}$ División, que ofrece muy buenas repeticiones detalles del juego.

En cuanto a las repeticiones, en este caso se utilizan tres magnetoscopios VTR, controlados por 2 operadores. El equipo técnico básico de la retransmisión lo componen:

Un director de imagen (equivalente en España al realizador) que también mezcla la señal de las cámaras (hace las funciones de mezclador), dos operadores de VTR, un operador técnico de sonido, un técnico de enlaces y un operador de control de cámaras. El partido es retransmitido por un narrador al que acompaña un comentarista. Ambos se encuentran en la mejor ubicación para transmitir el juego, una cabina central con una excelente visión del campo de juego. Otro reportero está abajo, en el césped, para llevar a cabo las entrevistas. Al igual que en todos los partidos de la Globo es importante reseñar la publicidad incluida en la narración del juego, al modo de "cuñas radiofónicas" y que el propio narrador

11 Partido Gremio -Intenalcional, retransmisión de Sportv (Globo), 7 Octubre 2000.

12 Sportv - Canal temático de deportes del grupo Globo nacido en 1991 cuya recepción es a través del sistema de pago Net/Sky. 
da paso a lo largo del desarrollo del partido con todos los tiempos marcados, elevando el tono de voz:

“...Premiere Esporte é la Copa Joao Havelange....Embratel,...”

Otros partidos de la Globo, que son en abierto, suelen hacerse empleando entre 10 y 12 cámaras, especialmente cuando son retransmitidos para todo Brasil en abierto llegando hasta un máximo de 18 como puede ser una Final de un Campeonato o Copa, o un partido de la selección brasileña.

\section{UBICACIÓN DE CÁMARAS: \\ PARTIDO DE COPA JOAO HAVELANGE \\ GREMIO-INTERNACIONAL \\ SPORTV (GLOBO)}

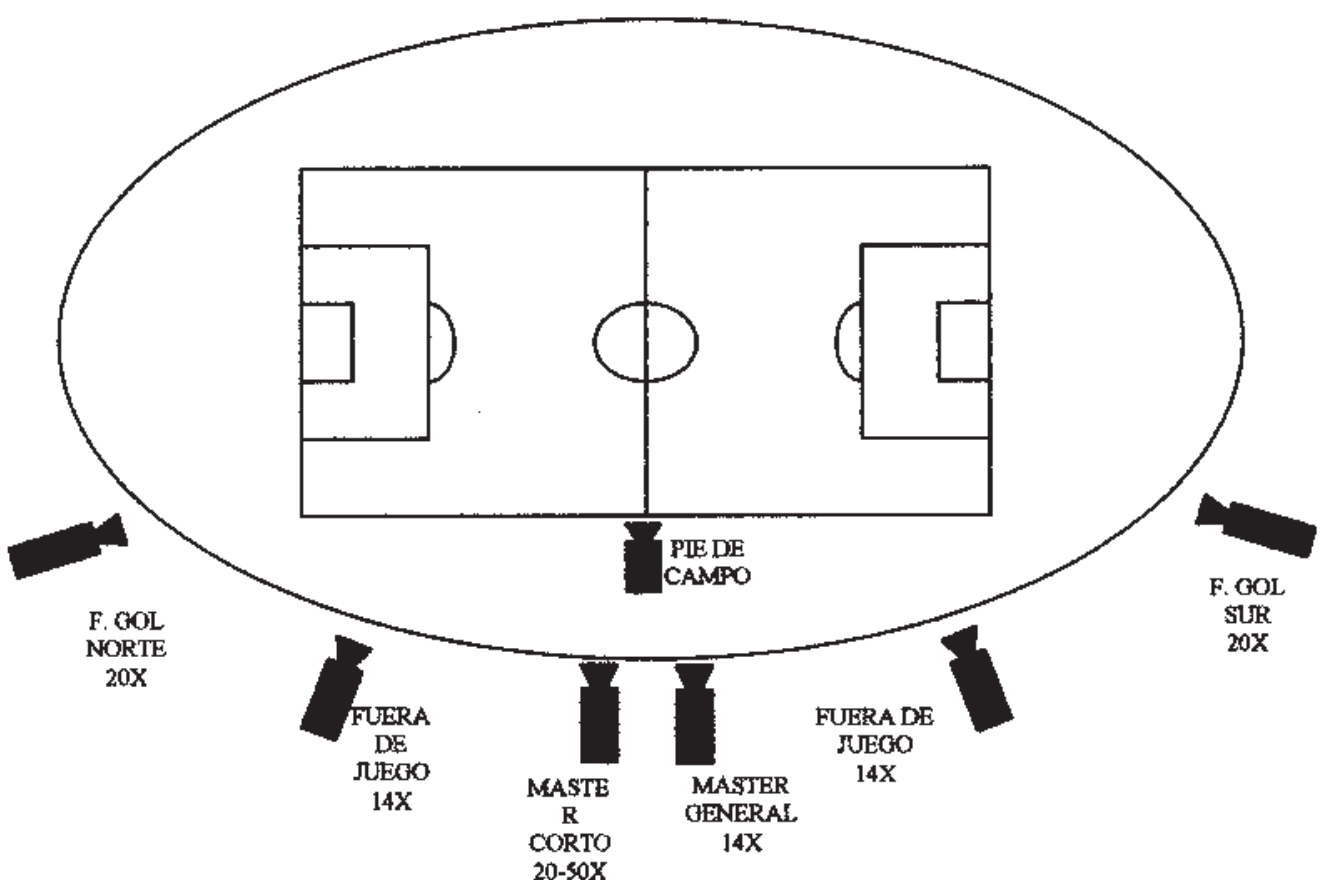


Es evidente que el fútbol se ha convertido en algo más que un simple juego o deporte, transpasando las más variadas culturas. En algunas, como la brasileña, pesa aún más, llegando incluso a convertirse en parte de su identidad cultural y nacional. Las emociones y sentimientos que genera lo que originariamente era un simple juego con un balón nacido en Inglaterra, se ha transformado con el cambio de siglo en una nueva industria, especialmente soportada por la televisión y el marketing deportivo. El hasta ahora conocido como aficionado (torcedor en Brasil) al fútbol, se transforma en el principal consumidor de esta nueva industria, cuya rentabilidad se basa en lograr la "felicidad" del aficionado.

La retransmisión de un partido de fútbol es un fiel reflejo de este fenómeno. Suelen ser los programas más vistos en televisión, especialmente por emitirse en horarios de máxima audiencia. De ahí que las diferentes cadenas la cuiden, sobre todo para ofrecer un evento deportivo lo más completo posible. Frente a la espectacularidad que en los últimos años están dominando las retransmisiones deportivas, Canal Sur ha preferido alejarse de esta "moda audiovisual" y centrarse más en el juego en sí. Se prefiere apostar por una producción y realización completa pero sin llegar al barroquismo de otras cadenas. El espectador asiduo a partidos de fútbol prefiere más ese plano master que le da la imagen del juego frente a planos más cortos que fracturan en demasía el evento deportivo. El significado del fútbol en Brasil como algo propio de su cultura y su pasión se observa también en su tratamiento en televisión. La cadena $R B S$ y el grupo $G L O B O$ cubren casi la totalidad de los principales partidos en el Sur del país y todo Brasil. La narración de los partidos, mucho más viva que en Canal Sur y la mayoría de las televiones de España, refleja claramente esta "pasión enfervorizada" que se puede ver en los propios profesionales de la información deportiva de Brasil. En este sentido, la cobertura de televisión es especialmente cuidada, con un estilo propio ( "patrón Globo" ) que busca transladar en la medida de lo posible el clima de todo el universo que rodea a un partido de fútbol. 


\section{Anexo}

EMISORAS DE RBS TV - SUR DE BRASIL
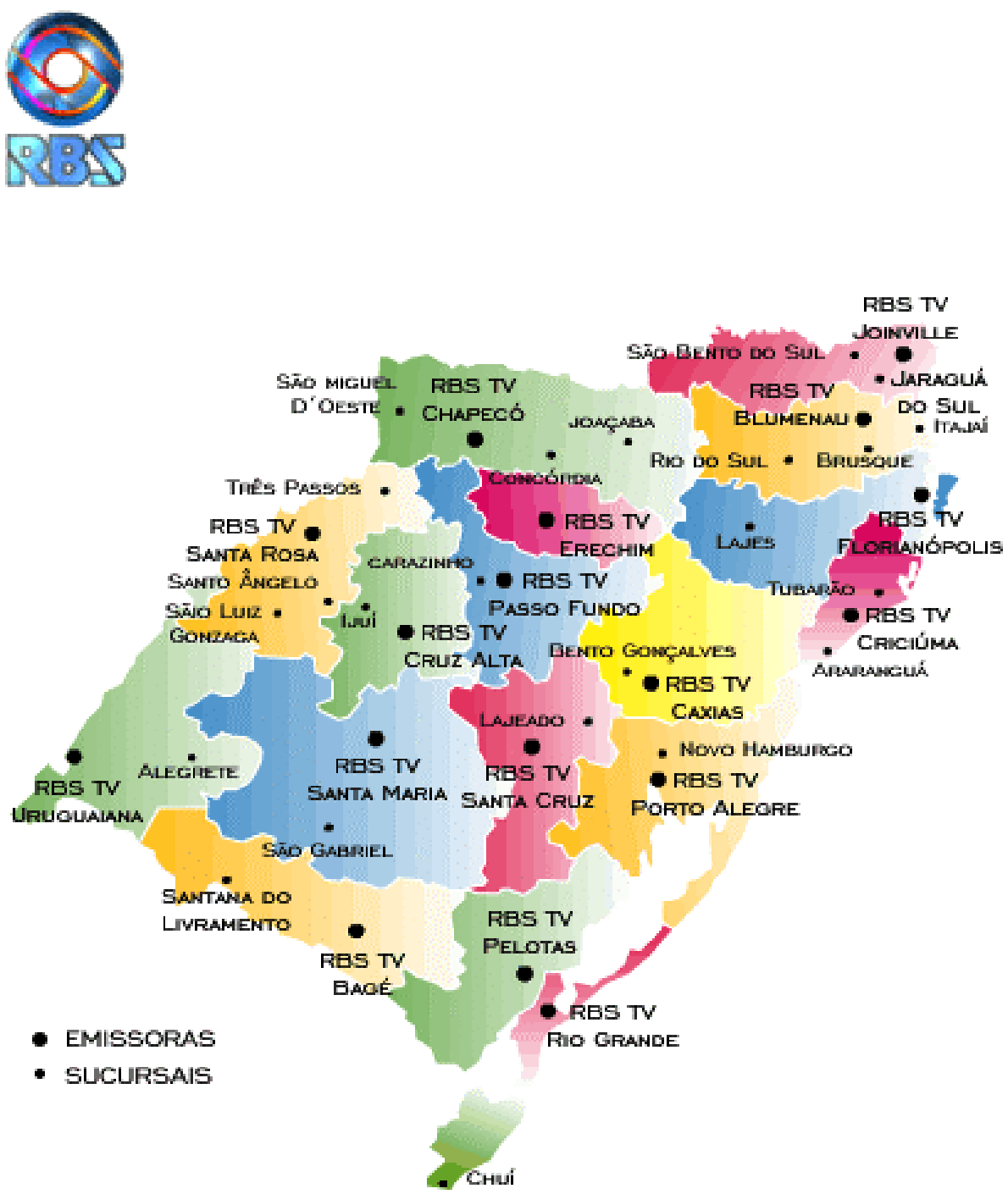


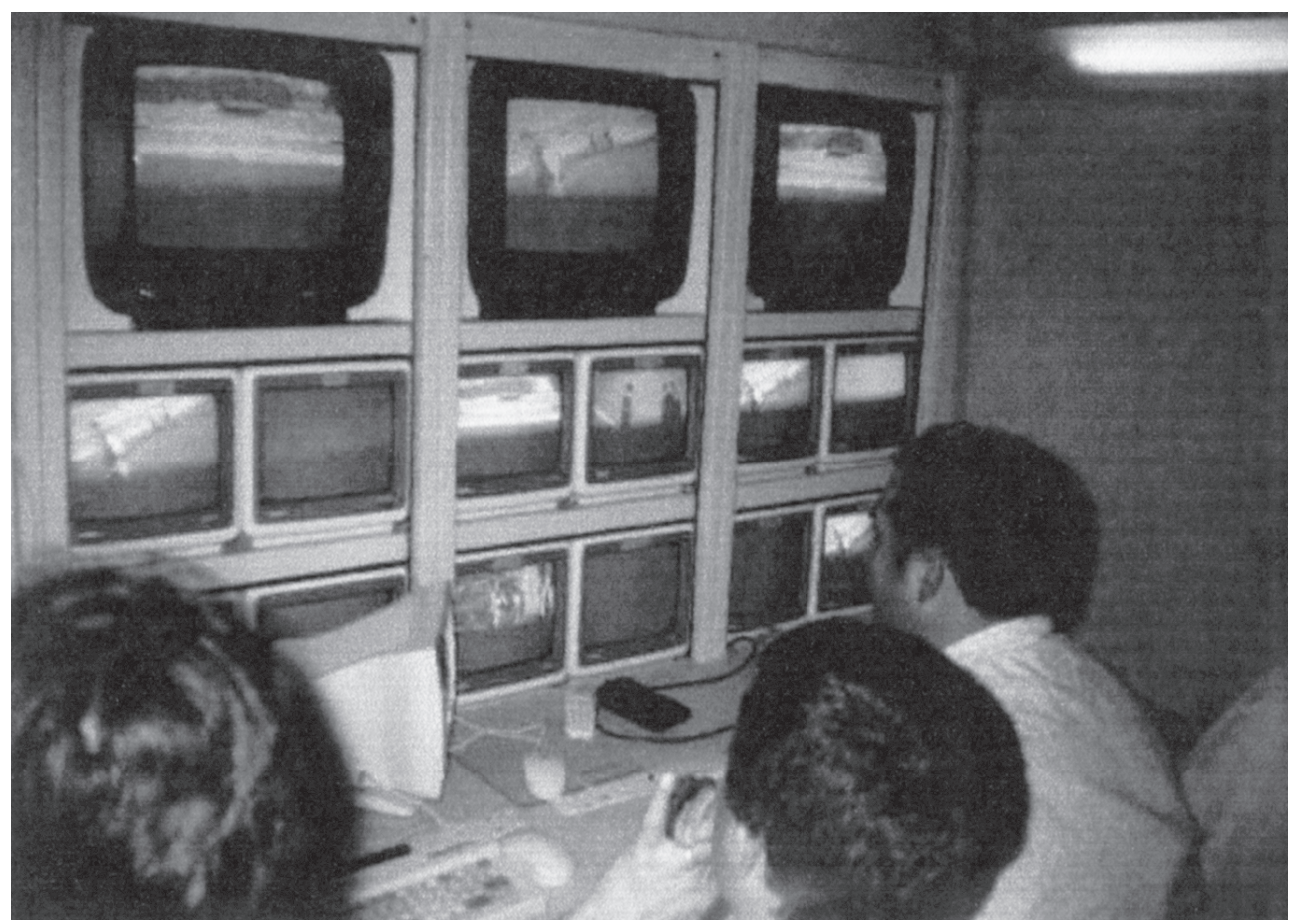

Control de realización de la U.Móvil de SPORTV 


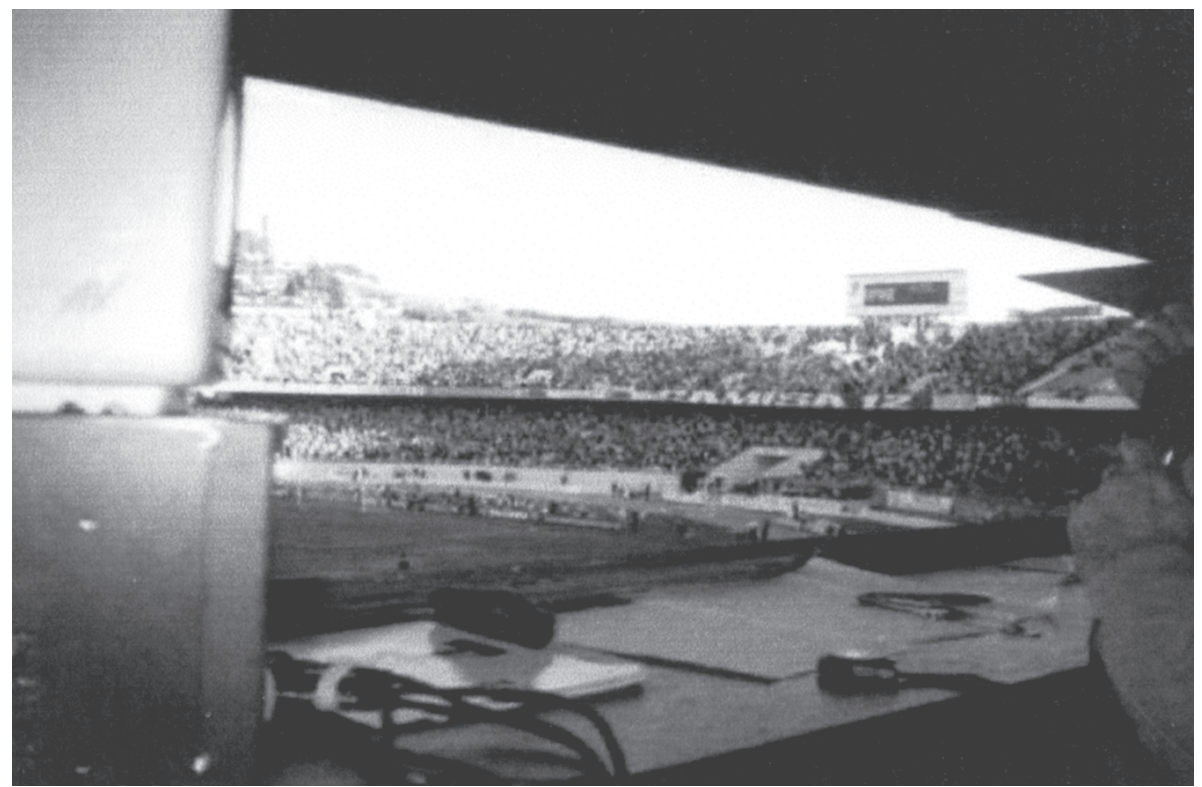

Cabina de comentaristas de $G L O B O$ Partido Gremio - Internacional

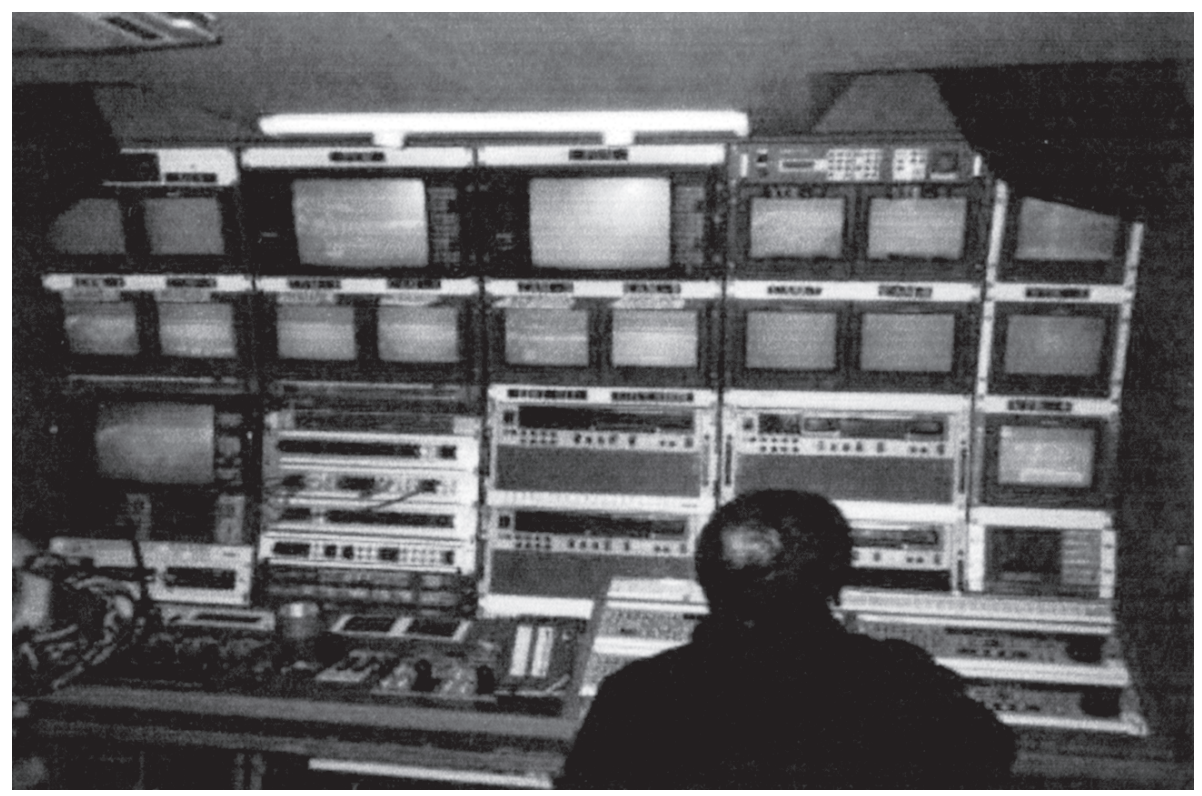

Control de realización de la U.Móvil de Canal Sur

(Recibido el 5-3-2001, aceptado el 20-3-2001) 